\title{
Design of Muscle Reflex Control for Human Upright Standing Push- recovery based on Series Elastic Actuator
}

\author{
Yuyang Cao, Kui Xiang, Biwei Tang, Zhaojie Ju, Muye Pang, Member, IEEE
}

\begin{abstract}
Ankle joint muscle reflex control plays an important role in the human biped posture. With the neuromuscular control mechanism and muscle-tendon biomechanical dynamic properties, our joints exnibit excellent performance in external environment physical interaction. Researching and understanding the mechanism of muscle reflex not only helps to reveal human movement control rules, but also provides a useful tool for robotic system design. In this paper, an ankle joint muscle reflex control strategy for human upright standing massage recovery is proposed. In order to focus attention on muscle reflexes and exclude other control mechanisms from the central nervous system (CNS) as much as possible, only ankle joint strategy without foot tilting is evoked by a limited external disturbance. A proportional derivative-like (PD-like) muscle reflex control algorithm is presented to understand the massage recovery of the human body standing upright. A series elastic actuator (SEA) based mechanical ankle joint is designed as a platform for testing activities. The experimental results show that the proposed reflection control method can achieve the upright standing push. Under the control of muscle reflex, the ankle joint behaves like a spring-damping system. Under such a simple and effective control strategy, it is easy to achieve upright station push recovery.
\end{abstract}

\section{INTRODUCTION}

Human upright standing is not an easy task because human center of mass is high above ground within relative small supporting feet. The human ankle joint plays an important role in upright standing balance, whose performance is pledged by neuromuscular control mechanism and muscle-tendon biomechanical dynamic properties. Muscle reflexes, as basic control loop, guarantee muscles steadily active response to external disturbance and enhance muscle dynamic properties [19]. studying the muscle reflex control on human upright push-recovery will not only highlight the reflex contributions on ankle joint dynamic properties but also provide an intelligent basic control method for robotic devices, such as exoskeleton devices and prosthetics.

Muscle reflexes, such as stretch reflex, act on the bottom frame of the control system, which is somewhat independent of the involvement of the cortex. It has a relatively short control loop, so the muscles can react very quickly to the disturbance. Faced with subtle disturbances in the

\footnotetext{
*Resrach supported by the National Natural Science Foundation of China under Grant [61603284]

Yuyang Cao is with the Intelligent System Research Institute, School of Automation, Wuhan University of Technology, China and Intelligent System \& Biomedical Robotics Group, School of Computing, University of Portsmouth, UK (phone: 759-789-4325; e-mail: 1287356777@qq.com)

Kui Xiang is with the Intelligent System Research Institute, School of Automation, Wuhan University of Technology, China.
}

environment, humans can keep their bodies upright through muscle reflexes alone. In the neuromuscular control mechanism, not only the body sway amplitude is an important input of the reflex control loop during upright standing push-recovery, but also the body sway velocity has a significant effect on the ankle extensor activity [1], because it carries about the body follow-up status information. In addition, the feedforward mechanism plays an important role in changing the reflection gain. The ankle joint can behave from very stiff to very compliant, allowing it to adapt to the environment. Some of them associate the reflex gain with the level of muscle activity, while muscle activity The level can be calculated from the myoelectric signal on the muscle surface. With in-depth biomechanical studies of ankle joint activity, some researchers have attempted to describe this behavior of the ankle joint through a specific control mechanism. In 1998, as a preliminary study, A relatively simple erect attitude balance stiffness control method [3] is proposed by Winter et al. This strategy assumes that the muscle acts as a spring that produces a restoring moment caused by the stiffness of the ankle joint in a quiet standing state. Two years later, in 2001, based on the biomechanical study of muscle ankle stiffness, they further proposed the muscle stiffness estimation of the elastic component of the ankle joint [4]. In 2006, Masani et al. proposed a feedback proportional derivative (PD) controller to ensure balance in a quiet posture. Even if the nerve delay is quite large, they provide evidence that the controller is effective. In 2008, focusing on interactive control, an assistance as needed (ANN) algorithm for promoting neural recovery is proposed in which the apparent mechanical impedance of the healing robot is programmable to a desired value to meet the adaptive requirements. In 2013, Through the impedance control inverted pendulum model and the bipedal walking model, the similarities and differences between the two concepts in these two concepts are elaborated [7]. These efforts have greatly assisted in the further study of ankle muscle reflex control.

This paper designs a SEA based mechanical ankle joint and proposes a specialized muscle reflex control for human upright standing push-recovery. The mechanical design of ankle joint is specified in Section II. The control strategy is elucidated hierarchically in Section III. The experimental

Biwei Tang is with the Intelligent System Research Institute, School of Automation, Wuhan University of Technology, China.

Zhaojie Ju is with the Intelligent System \& Biomedical Robotics Group, School of Computing, University of Portsmouth, UK

Muye Pang is with the Intelligent System Research Institute, School of Automation, Wuhan University of Technology, China. 
setup and process of experiment and analysis result are presented in Section IV.

\section{Mechanical Design of AnKle Joint}

In order to complete muscle reflex mechanism, a series elastic actuator (SEA) based mechanical ankle joint is designed according to human muscle-tendon unit, the structure of which exhibits significant viscosity [20]. The mechanical joint consists of foot and shank, shown in Fig. 1. The actuator, including motor and gear reducer, is fixed on the surface of mechanical shank and elastic element is placed inside the mechanical foot. The actuator drives mechanical foot rotation by cables and wheels inside the mechanical shank.

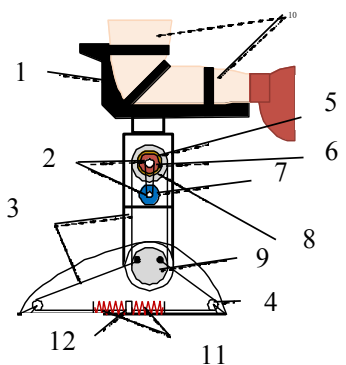

(a)

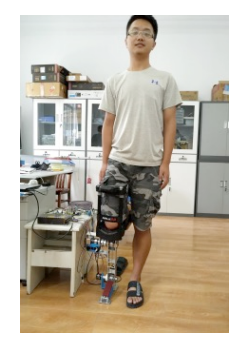

(c)
Figure 1. Mechanical design of ankle joint. (a) Structural schematic of ankle joint: (1) iWalk2.0 Hands Free Crutch, (2) belt pulley, (3) steel cables, (4) pulley, (5) coupling, (6) reducer, (7) motor, (8) wheel inside the shank, (9) wheel inside the foot, (10) experimenter's lower limb, (11) pressure springs, (12) slider. (b) Design of the SEA: Steel cables connect the springs to the actuator, which is detached from the ankle joint. (c) Photographic impression of SERAJ in operation.

\section{A. Series Elastic Structure}

Compared to traditional joint design, a elastic element [15] [16] is added between the actuator and load in mechanical ankle joint designed in this paper, guaranteeing joint's elastic behavior, to achieve compliant locomotion. Two pressure spring $(76.59 \mathrm{~N} / \mathrm{mm})$, as elastic elements, are placed inside the mechanical foot. Each spring is fixed at one end and another end of the spring can be compressed as the mechanical joint rotates. Direction of joint rotation decides which spring works and amplitude of joint rotation influences the spring compression:

$$
l_{s}=\left(\theta_{a}-\theta_{r}\right) R
$$

where $\theta_{a}$ and $\theta_{r}$ are rotation angles of ankle joint and gear reducer output shaft. $\mathrm{R}$ is the radius of wheel inside the mechanical shank, whose value is $0.05 \mathrm{~m}$.

\section{B. Separate Structure}

The ability of weakening negative influence of actuator's physical properties, especially it's mass and volume, on locomotion by algorithm is finite [6] that the separate structure is essential. Not only is cable drive used as an alternative of rigid connecting-rod but also actuator is fixed on the surface of mechanical shank, far from ankle joint, shown in Fig. 2.

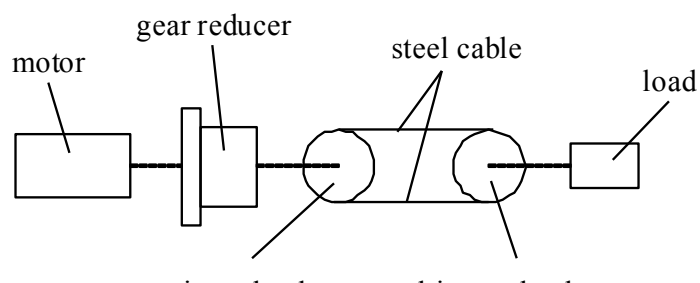

action wheel

driven wheel

Figure 2. the diagram of separate structure

\section{Muscle Reflex Control Strategy}

In this paper, the proposed muscle reflex control is derived from impedance control. Considering more muscle dynamic properties, a proportional-derivative-like (PD-like) muscle reflex control algorithm is given for better presentation of neuromuscular control mechanism.

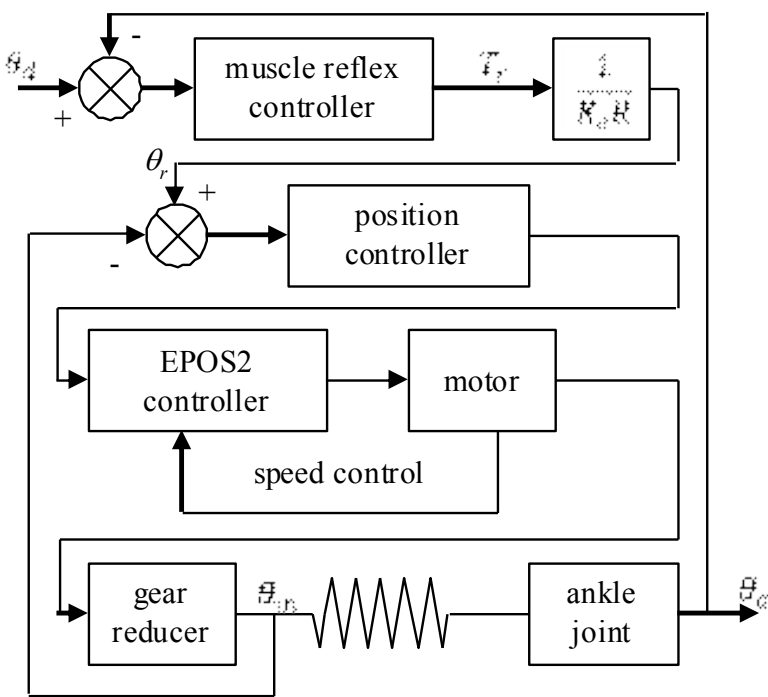

Figure 3. Diagram of ankle joint muscle reflex control

\section{A. Reflex Control Loop}

As a preliminary study, ankle joint is simplified as a single-degree-of-freedom joint in sagittal plane and subtle movements of other joints are ignored during human upright standing push-recovery. When people lean forward or backward, gravity creates a torque at the ankle joint due to center of mass shift. The torque created by gravity can be calculated by

$$
T_{G}=m g L \sin \theta
$$

Where $\mathrm{L}$ is the distance from center of mass to ankle joint and $\theta$ is the angle from center of mass to vertical direction.

When $\theta$ ranges from $-0.05 \mathrm{rad}$ to $0.05 \mathrm{rad}$, the torque created by gravity can be approximate given by

$$
T_{G}=m g L \theta
$$

So the torque created by gravity is thought proportional to center of mass shift during upright standing push-recovery.

In order to maintain standing balance, a reverse torque appears under muscle reflex control mechanism. The ankle joint stiffness is used to build the reverse torque-center of 
mass relationship [3][4]. Meanwhile, body sway velocity also have rich information about movement in the following time instant, which should be an adding input to reflex control.

$$
T_{r}=K_{s} \theta_{a}+D \dot{\theta}_{a}
$$

where $T_{r}$ is reserve torque. $K_{s}$ is stiffness of ankle joint. D is gain coefficient.

\section{B. Torque Control Loop}

As an advantage of SEA, torque control problem can be turned into position control problem, which improves the accuracy of control. According to mechanical design of ankle joint introduced in section II, the ankle joint torque can be expressed by

$$
T_{r}=K_{e} l_{s} R
$$

Where $K_{e}$ is spring constant, whose value is $76.59 \mathrm{~N} / \mathrm{mm}$.

\section{Speed Control Loop}

A maxon RE motor is chosen as driving motor so a maxon controller is used to achieve motor speed control. It can guarantee motor outstanding performance in this study.

\section{EXPERIMENTAL RESULT AND DISCUSSION}

In order to focus on muscle reflexes, controlling the experimental environment is considered to be pivotal $\{10\}$. in this study, "Ball-disturbance" is adopted as external disturbance during human upright standing push-recovery. When subject is in quiet stance, a rubber ball falls down from a height and hits the subject on the back, where muscle reflex control will appears, shown in Fig. 4. The mass of the ball can be adjusted to ensure subject can maintain standing balance under muscle reflex control.

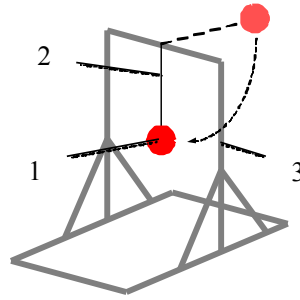

(a)

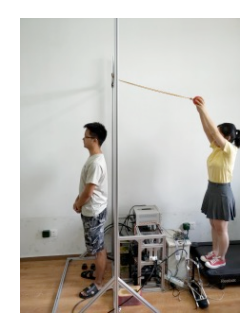

(b)

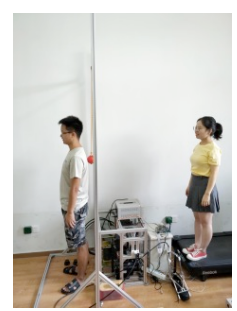

Figure 4. Experimental setup. (a) Structural schematic of experimental setup: (1) rubber ball, (2) connecting rope, (3) aluminum frame. (b) Photographic impression of experimental setup in operation.

According to the size of mechanical ankle joint, the specific subject ( $175 \mathrm{~cm}$ height and $65 \mathrm{~kg}$ weight) is selected to experience the "Ball-disturbance", equipped with mechanical ankle joint on his right knee by iWalk2.0 Hands Free Crutch, to prove the effectiveness of proposed control strategy and stiffness of ankle joint is given as $300 \mathrm{Nm} / \mathrm{rad}$. The subject is impacted by the ball falling down from $2.5 \mathrm{~m}$ high on the back. After light forward lean, the subject keep himself steadily upright. During the whole upright standing push-recovery, all real-time data is collected to display the real time-wave for intuitively describing ankle movement versus sway angle.

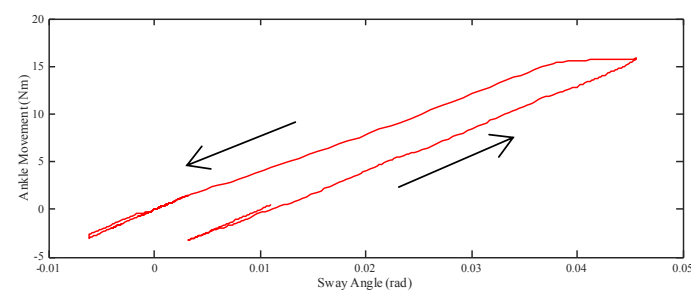

Figure 5. Ankle movement versus sway angle

The experimental result is shown in Fig. 5. At the beginning of experiment, the subject leans forward and reach the maximum sway angle $0.047 \mathrm{rad}$. In the whole deviation process, ankle movement created by mechanical ankle joint is basically linear with body sway angle under steady state. When sway angle reach the maximum value, the subject leans backward to maintain standing balance, where the ankle movement also shows linear change with sway angle and gradient is about $300 \mathrm{Nm} / \mathrm{rad}$. It is obvious that ankle movement in the recovery process was significantly greater than the deviation process at the same angle. This is because when the body resumes upright standing, the extra energy is provided with the spring energy, which is stored in the deviation process. The result is in agreement with human ankle joint dynamic property and the proposed control strategy is reasonable and feasible.

\section{CONCLUSION}

In this paper, a SEA based mechanical ankle joint is designed and a specialized muscle reflex control for upright standing push-recovery is proposed. The Ball-disturbance experimental result shows mechanical ankle joint has outstanding dynamic properties under muscle reflex control and upright standing push-recovery can be easily realized. In the future, the following two issues will be studied: 1) surface electromyography (sEMG) contains rich information for decoding motion intention in real time, which can be a useful tool in research on upright standing push-recovery; 2) stiffness of ankle joint is not constant and we need suitable method to measure it.

\section{ACKNOWLEDGMENT}

This work was supported by grant of the National Natural Science Foundation of China, the Excellent Dissertation Cultivation Funds of Wuhan University of Technology and the EU Seventh Framework Programme (Grant No. 611391).

\section{REFERENCES}

[1] Masani, K., Popovic, M. R., Nakazawa, K., Kouzaki, M., \& Nozaki, D. (2003). Importance of body sway velocity information in controlling ankle extensor activities during quiet stance. Journal of Neurophysiology.W.-K. Chen, Linear Networks and Systems (Book style). Belmont, CA: Wadsworth, 1993, pp. 123-135.

[2] Prochazka, A., Gillard, D., \& Bennett, D. J. (1997). Implications of positive feedback in the control of movement. Journal of neurophysiology, 77(6), 3237-3251.

[3] Winter, D. A., Patla, A. E., Prince, F., Ishac, M., \& Gielo-Perczak, K. (1998). Stiffness control of balance in quiet standing. Journal of neurophysiology, 80(3), 1211-1221. 
[4] Winter, D. A., Patla, A. E., Rietdyk, S., \& Ishac, M. G. (2001). Ankle muscle stiffness in the control of balance during quiet standing. Journal of neurophysiology, 85(6), 2630-2633.

[5] Masani, K., Vette, A. H., \& Popovic, M. R. (2006). Controlling balance during quiet standing: proportional and derivative controller generates preceding motor command to body sway position observed in experiments. Gait \& posture, 23(2), 164-172.

[6] Vallery, H., Veneman, J., Van Asseldonk, E., Ekkelenkamp, R., Buss, M., \& Van Der Kooij, H. (2008). Compliant actuation of rehabilitation robots. IEEE Robotics \& Automation Magazine, 15(3), 60-69.

[7] Rouse, E. J., Gregg, R. D., Hargrove, L. J., \& Sensinger, J. W. (2013). The difference between stiffness and quasi-stiffness in the context of biomechanical modeling. IEEE Transactions on Biomedical Engineering, 60(2), 562-568.

[8] Fitzpatrick, R., Burke, D. A. V. I. D., \& Gandevia, S. C. (1996). Loop gain of reflexes controlling human standing measured with the use of postural and vestibular disturbances. Journal of neurophysiology, 76(6), 3994-4008.

[9] Peterka, R. J. (2002). Sensorimotor integration in human postural control. Journal of neurophysiology, 88(3), 1097-1118.

[10] Jacobs, J. V., \& Horak, F. B. (2007). Cortical control of postural responses. Journal of neural transmission, 114(10), 1339.

[11] Buchanan, T. S., Lloyd, D. G., Manal, K., \& Besier, T. F. (2005). Estimation of muscle forces and joint moments using a forwardinverse dynamics model. Medicine \& Science in Sports \& Exercise, 37(11), 1911-1916.

[12] Buchanan, T. S., Lloyd, D. G., Manal, K., \& Besier, T. F. (2004). Neuromusculoskeletal modeling: estimation of muscle forces and joint moments and movements from measurements of neural command. Journal of applied biomechanics, 20(4), 367-395.

[13] https://simtkconfluence.stanford.edu/display/OpenSim/Musculoskeletal+Models \#MusculoskeletalModels-OpenSimCoreModels

[14] Hansen, N. (2016). The CMA evolution strategy: A tutorial. arXiv preprint arXiv: 1604.00772 .

[15] Cherelle, P., Matthys, A., Grosu, V., Vanderborght, B., \& Lefeber, D. (2012, June). The amp-foot 2.0: Mimicking intact ankle behavior with a powered transtibial prosthesis. In 2012 4th IEEE RAS \& EMBS International Conference on Biomedical Robotics and Biomechatronics (BioRob) (pp. 544-549). IEEE.

[16] Pratt, G. A., \& Williamson, M. M. (1995, August). Series elastic actuators. In Proceedings 1995 IEEE/RSJ International Conference on Intelligent Robots and Systems. Human Robot Interaction and Cooperative Robots (Vol. 1, pp. 399-406). IEEE.

[17] Pang M, Li M, Xiang K. (2017). Human ankle joint reflex and impedance control during upright stance balance. Journal of Huazhong University of Science \& Technology, 45(10):49-53.

[18] Au, S. K., Herr, H., Weber, J., \& Martinez-Villalpando, E. C. (2007, August). Powered ankle-foot prosthesis for the improvement of amputee ambulation. In 2007 29th annual international conference of the IEEE engineering in medicine and biology society (pp. 30203026). IEEE.

[19] JOHNSTON, \& I., A. . (1984). Muscles and movement: muscles, reflexes, and locomotion. Science, 226(4680), 1308-1308.

[20] Roberts, T. J. . (2002). The integrated function of muscles and tendons during locomotion. Comp Biochem Physiol A Mol Integr Physiol, 133(4), 1087-1099. 